Article

\title{
Does Fear of Isolation Disappear Online? Attention-Seeking Motivators in Online Political Engagement
}

\author{
KyuJin Shim ${ }^{1, *}$ and Klive (Soo-Kwang) Oh ${ }^{2}$ \\ ${ }^{1}$ School of Culture and Communication, University of Melbourne, Parkville, VIC 3010, Australia; \\ E-Mail: kyujin.shim@unimelb.edu.au \\ ${ }^{2}$ Communication Division, Pepperdine Seaver College, Malibu, CA 90263, USA; E-Mail: klive.oh@pepperdine.edu \\ * Corresponding author
}

Submitted: 30 September 2018 | Accepted: 11 November 2018 | Published: 19 February 2019

\begin{abstract}
This study investigated the effects of fear of isolation (FOI) on political content consumption and creation in the context of online communication. Using more than 1,000 respondents from South Korea, the study empirically tested a theoretical model of FOI on political content consumption and expressions with two mediators (i.e., attention/status-seeking, and anonymity-seeking). Results indicated that FOI is related to seeking attention and status in political outlets also connected to anonymity-preference that leads to political expression. Implications for political communication scholarship and for practitioners are that voters' political participations can be understood in a framework different from traditional focus on persuasion, political ideology, or demographics because-in today's virtual and interactive media environment-users are more content consumers or community participants.
\end{abstract}

\section{Keywords}

attention-seeking; anonymity-seeking; fear of isolation; political communication; social media; status-seeking

\section{Issue}

This article is part of the issue "Emerging Technologies in Journalism and Media: International Perspectives on Their Nature and Impact", edited by John Pavlik (Rutgers University, USA).

(C) 2019 by the authors; licensee Cogitatio (Lisbon, Portugal). This article is licensed under a Creative Commons Attribution 4.0 International License (CC BY).

\section{Introduction}

On March 10, 2017, South Korea's Constitutional Court unanimously decided in favor of the impeachment of then-President Park Geun-hye. Her involvement in corruption and scandalous ties to her aides had sparked national uproar, fostering an unprecedented level of political participation in which, inter alia, the public gathered en masse on streets and in public squares for candlelight vigil protests, in unprecedented numbers (Lim, 2016). However, another notable phenomenon could be found in online news consumption and discourse. One conspicuous distinction was that on Korea's top news portal site, Naver News, relevant articles in the politics section were the most viewed story and received the most comments for every day of 2017. In fact, one story aloneon March 10 -received in excess of 27,000 comments
(Naver, 2017). One of the highest-commented news articles ever was about former president Lee Myung-bak's controversial remarks regarding the island of Dokdo, which surpassed 349,000 comments (Kim, 2010). Considering that the website limits users to 20 comments per day, this level of participation is significant. As for other online platforms, eight of the top ten most listened-to podcasts are about politics (Podbbang, 2017).

South Korea has been considered a frontrunner in terms of online news consumption (Reuters, 2016), owing to the popularity of news portal services that aggregate numerous news outlets in a single website. Coupled with advances in mobile technologies, users conveniently can access news stories and instantaneously comment on them, vitalizing political discourse online (Houston, Hansen, \& Nisbett, 2011, p. 79). This is particularly associated with news presentation/consumption 
trends in South Korea. Since the early 2000s, South Korea's digital media landscape has been coupled with internet portal websites, the big three being Naver, Daum and Nate. Each of these began with personal media services (e.g., email and personal profile pages), developing their services into web search and news consumption. In fact, recent reports rank the so-called internet/news portals among the top five websites visited by Korean users (Chang Center Group, 2017; Nielsen KoreanClick, 2018). These portal platforms have become a prominent news aggregator, housing news articles from mainstream newspapers and broadcast stations to onlineonly sources as well as alternative media outlets. Each website features a news-commenting section unique to each portal, and these have become the arena and barometer for political opinions and engagement, as shown in Naver's record-breaking user-commenting activity above.

In the Western world, political discourse occurs most often and actively on social media platforms (e.g., Facebook and Twitter) (Enli, 2017; Ott, 2017). However, Korea's unique characteristic of news consumption and engagement makes portal-based news commenting a key aspect of political engagement in the country, thus warranting a closer look.

This study explores online users' motives for political participation from the theoretical frameworks of fear of isolation (FOI) and attention-seeking, which may lead to political disinhibition (Suler, 2004) - that is, increased political participation online. We posit that individuals who display FOI due to their political opinions will use online platforms actively for their attention-seeking and anonymity-seeking preferences. Being able to gauge the political climate and express opinions anonymously will reinforce those individuals' willingness to be recognized and to be influential, tendencies that could augment political online participation and expression.

We believe this study explores a new framework of political psychology that goes beyond conventional paradigms (e.g., selective exposure based on partisanship or ideology) and contributes to a better understanding of anonymous and attention-seeking online behaviors in politics-based news consumption and conversation. We hope to propose a new definition of individuals' "preference of online" with emphasis on online efficacy and motivation/gratification factors sought after in the online sphere.

\section{Literature Review: Fear of Isolation and Factors for Online Political News Consumption and Discourse}

FOI is defined as a "psychological variable representing a negative emotional state associated with the prospect of voicing one's opinion about a given topic." (Neuwirth, Frederick, \& Mayo, 2007, p. 452). FOI is associated with perceived public opinion-when others seemingly disagree with an individual's opinion on a topic matter, that individual feels discouraged from sharing his or her own
(Noelle-Neumann, 1974). Also, as discussed in Neuwirth et al. (2007), the theoretical framework of communication apprehension is applicable to FOl; perceived disagreement of the self's opinions with the group's may cause "fear or anxiety associated with either real or anticipated communication with a person or persons" (pp. 452-453). Pertinent literature on FOI associate it with the spiral of silence, indicating that FOI often leads to silence or conformity to the perceived dominant opinion. The same applies to the online environment-in Gearhard and Zhang (2015), agreeable political content was a predictor for the willingness to speak out, while exposure to disagreeing postings hindered opinion expression. Moreover, findings on motives for selective posting (Porten-Cheé \& Eilders, 2015; Yun \& Park, 2011) imply that online participation increases as people look for homogeneity of opinions.

Based on the above, FOI mostly has been linked to bandwagon effects or to explain successful agendasetting by mainstream media (Liu \& Fahmy, 2011). However, we focus on a different potential outcome related to FOI: increased online activities. This might seem counter to the concept of fear, which implies discouragement. However, we posit that FOI may well lead to increased political activities, especially given the characteristics of the online environment.

Reasons for our argument are threefold: first, FOI has to do with the need for selective exposure to avoid situation where one's ideas are likely to be rejected by others. We have seen increasingly-partisan positions of media, and people easily filter content based on perceived ideological congruence (Bennett \& Iyengar, 2008; Coe et al., 2008; lyengar \& Hahn, 2009). And we note that the online sphere encourages 'partisan media' which enhance selective exposure via increasing capability of selfgatekeeping. Much of the literature has focused on polarization and selective exposure online, as online enables users to choose stories from different sources based on political heterogeneity (Messing \& Westwood, 2014). So this trend enhances partisan affiliation that helps relieve fear of isolation, given its capacity to avoid or bypass real-life encounters in political discrepancies. Ideas FOI seeks willingness to voice bypass real-life penalties and sanctions incurred from being objective against dominant opinions. This is somewhat contradictory of classic FOI theory which indicates connection between FOI and spiral-of-silence to avoid sanctions and penalties.

Also, developments in speed and interactivity have allowed for a facilitated access to information and ideas (Jensen, 2017) which makes it easier for an individual to determine, from her or his perspective, what the dominant public opinion seems to be (Springer, Engelmann, \& Pfaffinger, 2015). This conjecture is based on the fact that an individual thus choose from multiple platforms while focusing on sites offering opinions consistent with her/his view, and can ignore sites offering alternative views.

Second, as a result of this increased degree of FOI, individuals might be inclined to constantly consume on- 
line political content (news, user posts, comments, etc.) to gauge the public opinion climate. This might be accelerated among those with FOI, since these individuals worry that their opinions might conflict with dominant ones (Hayes, Matthes, \& Eveland, 2011; Kim, Kim, \& Oh, 2014; Weiman et al., 2012).

Finally, the online environment allows for individuals with FOI to do something about their fear. While in past studies FOI has been associated most closely with spiral of silence, we posit that it might actually lead to increased participation in online political discourse. In the online setting this is possible because of anonymity, inclusivity and virtual identities (Nagy \& Koles, 2014). Masked by an online identity, an individual need not experience isolation in real life-rather, the individual might utilize interactive features online to easily post ideas conforming to the (perceived) dominant opinion. For those with FOI, we argue that this increased activity may be a way to react to their fears and ultimately resolve them by being a part of the conversation (and not being isolated). By the same token, consequences of online communication are lighter, meaning there is a smaller chance of the individual facing actual isolation as may opposed to when engaging in face-to-face communication. This is further discussed below in light of a possible anonymity-seeking tendency among individuals with FOI.

\section{Attention-Seeking and Online Political News Consumption and Discourse}

Many studies have found that narcissism and subsequent attention and status-seeking are strongly connected to online behaviors (e.g., DeWall, Buffardi, Bonser \& Campbell, 2011; Huberman, Romero, \& Wu, 2009). It is predicted that narcissists communicate in ways that draw attention to themselves via profane and self-promotional content (DeWall et al., 2011). In the same vein, one study on YouTube video uploading and downloading behaviors has found that productivity in crowdsourcing is strongly affected by attention-seeking (Huberman et al., 2009). So it is indicated that attention is an important driver of contributions to the digital commons. Interestingly, participants' attention-seeking preference is affected by online content consumption behaviors exhibiting the aggregate bandwagon effect. This means that attentionseekers tend to appeal to the majority opinion in the online community which is mostly partisan media accommodating political interest for the party not the mass, so the bandwagon effect might emerge at community level in the context of digital communication. This is in contrast to the mass communication era. Especially, popularity cues on the viewership of online user-generated content stimulate both consumption and participation behaviors.

To note, "web users...overwhelmed by information and quality uncertainty, will gravitate toward the popular choices made by earlier decision makers, which appear via indicators such as hit counts to forge quality impressions" (Hu \& Sim, 2011, p. 2382). Moe and Schweidel
(2012) also found that positive ratings environments increase posting incidence, whereas negative ratings environments discourage posting. This finding indicates that political news consumption and conversation might be the consequence of inherent needs of attention, reputation and status and so on. Accordingly, it is conjectured that the traditional notion of the bandwagon effect should be understood in a novel light in the context of online communication. Bandwagon effect traditionally is based on an individual's need to avoid social isolation arising from discrepant opinions. And, with increasing interactivity in online communication, the attentionseeking preferences of users wanting to belong to major public opinion by making themselves part of a politicallyhomogeneous community, may drive the bandwagon effect. Applying this notion to the context of political news consumption and participation, we presume that attention-seeking behaviors will lead to politics-based news consumption and conversation.

\section{Anonymity-Seeking in Political Expression}

We posit that anonymity in the online environment will be an important factor for increased political activities among individuals with FOI. That is, as online media expand in their utility as tools for public opinion formation, isolation in real-life settings can be overcome with anonymity and interactivity in the online environment (McElroy, 2013; Nielsen, 2014). Communication factors such as anonymity, invisibility, and lack of face-to-face contact provide the opportunity of lowering behavioral inhibitions in online media environment (Joinson, 2003, 2007; Kiesler, Siegel, \& McGuire, 1984; Lapidot-Lefler \& Barak, 2012; Suler, 2004).

Anonymous conversations are unique to online communication (Berg, 2016). Anonymity enables an alter ego whereby one can build a hidden and virtual identity to possibly protect one's real identity from the potential threat of negative outcomes (e.g., criticism, disapproval, isolation) imposed on minority opinions. We note that anonymity helps users to relieve psychological discomfort and self-monitoring efforts when posting comments or expressing their opinions in the face of fear of potential negative outcomes (Bailenson, Yee, Merget, \& Schroeder, 2006). Indeed, anonymity was a factor that encouraged individuals to give opinions about controversial issues (Berg, 2016; Cho \& Kwon, 2015). Lack of faceto-face confrontations helps avoid heated discussions, therefore fostering an environment where participation from all individuals is welcome (Papacharissi, 2004). In fact, online anonymity has been found to decrease one's inhibition and to increase self-disclosure (Bailenson et al., 2006; Bargh, McKenna, \& Fitzsimons, 2002; Chiou, 2006, 2007; Joinson, 2001).

From the above, we further speculate that individuals with FOI will in fact display anonymity-seeking tendencies. In general, online users favor anonymous features in political conversations and engagement, be- 
cause anonymity in online news sites allows for an individual to voice opinions more freely; online aliases are not directly linked to an individual's own personal and/or professional networks (Nielson, 2014). Again, this is contrary to traditional schools of thought on FOI, which emphasize a spiral of silence-type behavior. However, given that online anonymous platforms enable people to do what they usually would not in the real world (Suler, 2004), we can posit that anonymity can free people from potential fear occasioned by active political engagement and expression.

As noted above, we conjecture that FOI would be associated with the need to be a part of a partisan community who share common political ideologies. Therefore, one could easily draw attention or status from peer members in the group through excessive and enthusiastic politics-based knowledge attainment and conversation. To be specific, online participation would be driven by status and status-seeking, and that status sentiments are more likely to sustain online communities (Lampel \& Bhalla, 2007). We would like to focus on self-serving interest as a major driver to participate in political conversations via online.

In sum, attention/status-seeking (DeWall et al., 2011; Lampel \& Bhalla, 2007) and anonymity-seeking can encourage people to do certain things that they would not usually do in the real world (Suler, 2004). Therefore, we hypothesize that such attitudinal and behavioral tendencies of FOI combined with characteristics of online media will amplify an individual's political consumption and participation online.

H1) FOI will be associated to a) attention/statusseeking and b) anonymity-seeking preferences online.

$\mathrm{H} 2$ ) a) Attention-seeking and b) anonymity-seeking preferences online will lead to politics-based news consumption and conversation.

H3) FOI will be indirectly related to politics-based news consumption and conversation via a) attention/ status-seeking and b) anonymity-seeking preference online.

\section{Method}

\subsection{Procedure}

A web-based survey was conducted to answer proposed research questions and test hypotheses. The survey was administered by the Korean panel of Macromill Embrain Co., a leading research firm. The survey was conducted from January to February 2017. A total of 1,107 respondents participated in the survey. The probability quota sampling was designed based on Korean population statistics (Ministry of the Interior, 2017). Participants from the general population were given credit according to the individual reward policy of the company. The number comprised of $47.8 \%$ female, $52.2 \%$ male. Average age of participants was 40.75 . We also excluded "less than 20" and "more than 60" age brackets as our study focused on the population of online users in the political context. However, it should be noted that both age groups are active online in the Korean political context, therefore, the sample might not represent Korean online-sphere demographics. Age group numbers are as follows: 228 in the 20-29 bracket (20.6\%), 280 in the 30-39 bracket, (23.4\%), 275 in the 40-49 bracket (24.7\%), 315 in the 50-59 bracket (28.5\%). Participants age- and gender-distribution was close to the regional population representation designed by our quota sampling. Compared to the statistics (excluding the "more than 60 " and "less than 20" age bracket), our sample is fewer in 20-29 (-0.4\%), 30-39 (-0.6\%), 40-49 (-3.3\%) and more $50-59(+1.5 \%)$. Given that Internet use is saturated among younger generations but not among older (Statista, 2016), this distribution is acceptable.

\subsection{Measures}

Most of the survey items were adapted from prevalidated research work, so as to increase the construct validity, except for the measure for anonymity-seeking and politics-based news consumption and conversation (PNCC), which were created for this study. For all measures, 5 point Likert scales were used ( $1=$ strongly agree, $3=$ neither agree nor disagree, $5=$ strongly disagree) except for PNCC which was measured with amount of time. All items for each variable are displayed in Appendix 1.

\subsubsection{Political News Consumption and Conversation Online (PNCC)}

A composite index of PNCC was created by adding a total of six items measuring the frequency of respondents' use of social media for political content. Six major social media platforms and portal site news platforms were selected based on their impact on the political landscape in South Korea. Respondents were asked to report their use in ten-minute intervals (from 0 to $60+, 1=0 \mathrm{~min}$, $8=$ more than $60 \mathrm{~min}$ ), i.e., how long they used social media for political news checking and participation in conversations (Range $=6$ to $48, M=16.34, S D=7.76$ ).

\subsubsection{Fear of Isolation (FOI)}

To measure FOI, we borrowed three items from Kim (2012). The items include "I worry about being negatively evaluated when others disagree with me", "I tend to let the other person win an argument even when I know I am right", and "If my political opinion were to become widely known around my workplace, I am very concerned that people would avoid me or act differently towards me" ( $\alpha=.64, M=2.82, S D=0.47)$.

\subsubsection{Attention/Status-Seeking}

We borrowed six measurement items to gauge perceived attention/status-seeking from Berger (2014). The items 
include "I want to be perceived positively", "I would like to look special", "I would like to be considered an expert", "I would like to be considered more knowledgeable", "I would like to be seen as an opinion leader" and "I would like to receive attention from others" ( $\alpha=.89$, $M=3.05, S D=0.72$ ).

\subsubsection{Anonymity-Seeking}

We developed four measurement items to gauge perceived anonymity-preference. The items include "During anonymous online conversation via social media or online news sites, I feel less constrained to talk about certain things than in conversations where I can be identified", "Anonymous online platforms enable me to support my favorite politicians on political-news websites", "With anonymity online, I can argue with someone of different opinions without reservations, in comparison to in the offline setting", and "Anonymous online platforms enable me to engage in political discussions" ( $\alpha=.87$, $M=3.30, S D=0.67)$.

\section{Results}

The study tested a structural model of attention/statusseeking and anonymity-seeking preferences as mediators between FOI and PNCC. Data were analyzed with the AMOS 23 software program, with a covariance-based ap- proach, using maximum-likelihood estimation. The structural equation model was developed after including all hypothesized paths among variables: $\chi^{2}(54, \mathrm{n}=1000)$ $=203.94 .64, \mathrm{p}<.001, \chi^{2} / \mathrm{df}=3.183, \mathrm{SRMR}=.0361$, $\mathrm{TLI}(\mathrm{NNFI})=.969, \mathrm{RMSEA}=.046$, and CFI $=.978$.

\subsection{Hypotheses Testing}

The suggested model (see Figure 1) indicated all hypothetical paths to be statistically significant. The effect of FOI on attention/status-seeking $(\mathrm{H} 1 \mathrm{a})$ was significant ( $\mathrm{B}=.334, \mathrm{~S} . \mathrm{E} .=.048, \beta=.431, \mathrm{p}<.001)$. The effect of FOl on anonymity-seeking ( $\mathrm{H} 1 \mathrm{~b})$ was significant $(\mathrm{B}=.730$, S.E. $=.070, \beta=.483, \mathrm{p}<.001)$. So $\mathrm{H} 1$ was confirmed statistically significant. The effect of attention/statusseeking on politics-based news consumption and conversation $(\mathrm{H} 2 \mathrm{a})$ was significant $(\mathrm{B}=2.294, \mathrm{~S} . \mathrm{E} .=.490$, $\beta=.162, \mathrm{p}<.001)$. Also, the effect of anonymity-seeking on politics-based news consumption and conversation $(\mathrm{H} 2 \mathrm{~b})$ was significant $(\mathrm{B}=1.847, \mathrm{~S} . \mathrm{E} .=.323, \beta=.186$, $\mathrm{p}<.001)$.

\subsection{Mediation Analysis}

H3 posited the mediating effect of key traits of social media such as attention/status-seeking. The indirect effects of FOI on PNCC via attention/status-seeking (H3a) and anonymity-seeking ( $\mathrm{H} 3 \mathrm{~b})$ were examined using an

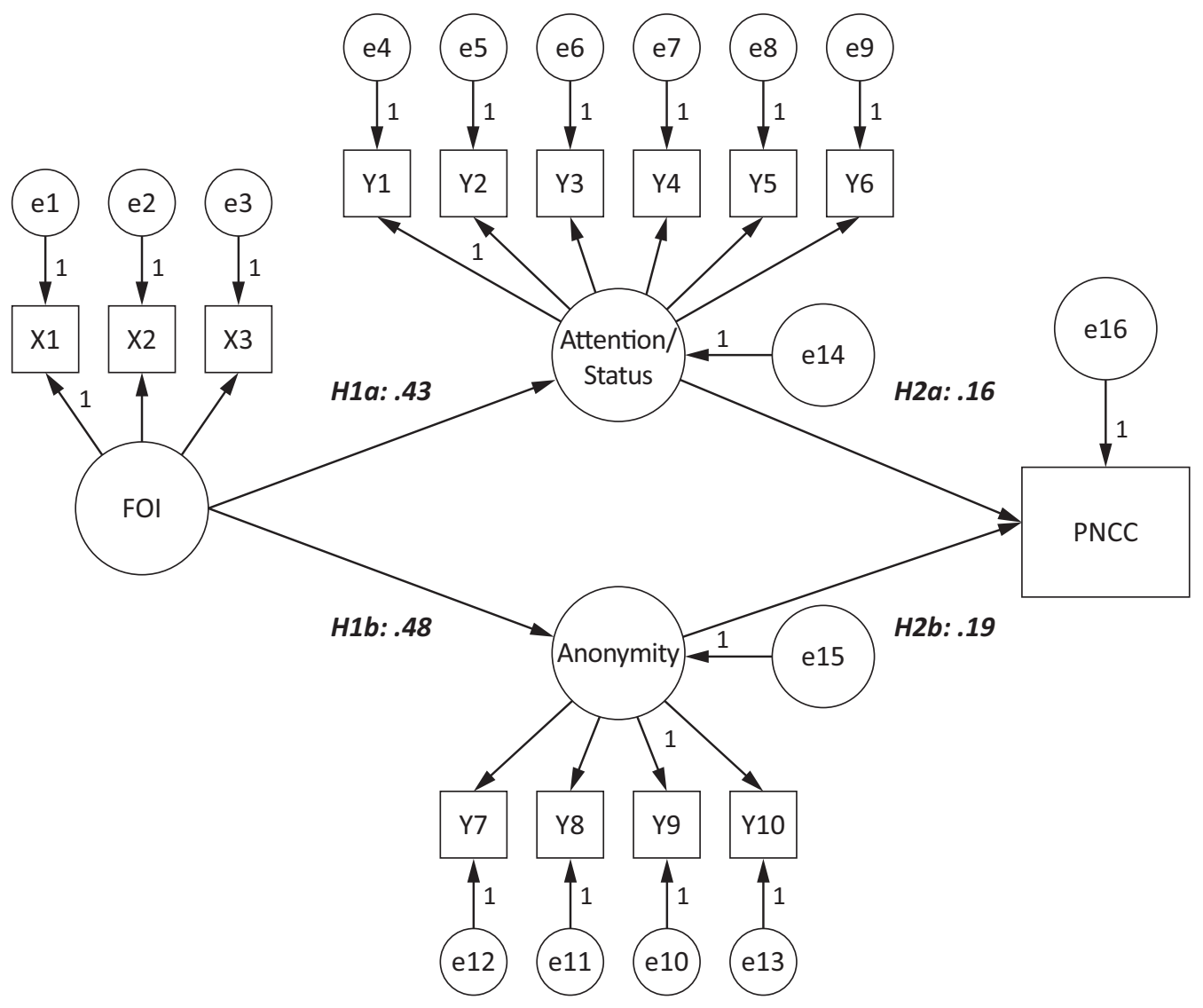

Figure 1. Results of testing hypotheses with standardized path coefficient. 
approach proposed by Baron and Kenny (1986), and the significance of the indirect effects was examined using bootstrapping techniques. As suggested by Preacher and Hayes (2008), 5,000 bootstrap samples were computed and a bias-corrected $95 \%$ confidence interval (Cl) calculated. H3a examined whether attention/status-seeking would connect FOI with PNCC. The indirect effect of FOI on PNCC via attention/status-seeking was significant ( $\beta$ [Bootstrap 95\% Cl] $=.044[.018, .067], \mathrm{p}<.005)$. And $\mathrm{H} 3 \mathrm{~b}$ examined whether anonymity-seeking would mediate between FOI and PNCC. The indirect effect of FOI on PNCC via anonymity-seeking was significant ( $\beta$ [Bootstrap $95 \% \mathrm{Cl}]=.049[.021, .073], \mathrm{p}<.005)$. Hence, $\mathrm{H} 3 \mathrm{a}$ and $\mathrm{H} 3 \mathrm{~b}$ all were supported.

\section{Discussion}

This article takes a different approach to $\mathrm{FOI}$ and suggests a new model for understanding its role for online political discourse. This model suggests that FOI is a possible factor for online activities such as news consumption and commenting. Traditionally, FOl has been studied in association with passive effects (e.g., bandwagon effect, agenda-setting). However, this study implies that FOI actually be a major predictor for active news engagement (news consumption, commenting) online.

Firstly, we found that FOI was associated with attention/status- and anonymity-seeking preferences online, and that these preferences led to politics-based news consumption and conversation. This means that the individual's fear resulted in an increased motivation to become part of the mainstream (dominant opinion), rather than be shut them down. Note that this correlation should be understood particularly in the context of online communication, as online features such as interactivity, anonymity and accessibility help create a virtual and loosely-knit yet politicaly- homogeneous community. This finding can drive future theorizing on FOI, anonymity, status-seeking and opinion congruency in relation to the "bandwagon" effect on the level of online community, which contradicts the previous notion of FOI and spiral-of-silence.

In fact, we found that stronger the FOI, the higher an individual's desire for attention and heightened status. And this finding is congruent with previous indication from Hayes et al. (2011) that "fear of social isolation (FSI) prompts people to seek out information about the climate of public opinion" (p. 439). That means, fear tends to enhance the need for sense of belonging to dominant opinions in the online context, thereby motivating people to act and participate to make dominant their own opinions. This leads fearful individuals to have a stronger urge to consume information (to comprehend what the dominant opinions are) and to become part of the discourse (through conversations in commenting threads) (Velasquez, 2012). So it is conjectured that they find likeminded people online who share their viewpoints and that this makes them feel less isolated.
Noteworthy here is that these tendencies are shown in the online environment, due to these individuals' anonymity-seeking behavior. Per H3, we found that FOI was related indirectly to politics-based news consumption and conversation via attention/status-seeking and anonymity-seeking preferences online. We must note that these individuals still have high levels of FOI, and the fear is most detrimental in possible face-to-face isolation and negative responses from others. Therefore, FOI individuals turn to online platforms where they can participate in conversation through virtual identities (Joinson, 2001; Lapidot-Lefler \& Barak, 2012;). This reduces anxiety about what might come from the consequences of their actions. Coupled with the attention/statusseeking characteristics, FOI individuals indeed become highly motivated to engage in political news consumption/conversation online. Other studies also found that willingness to gauge the public opinion climate was associated with the willingness to speak out (Nielsen, 2014; Yun \& Park, 2011), but the influence of anonymity per se in the online setting was not confirmed. In this light, this study contributes to scholarship through its exploration of the linkage among FOI, anonymity and the willingness to engage in political discourse.

Another contribution of this study to scholarship about online political behaviours is that it provides a theoretical framework of digital consumerism to understand political news consumptions and participations. While studies on marketing and virtual communities (e.g., DeWall et al., 2011) have focused on "attention/statusseeking" in relation to the motivation of content creators and community leaders' political communication, scholarship has paid little attention to this area. Considering that traditional political scholarship has examined participants mostly with close attention to political ideology or orientation, partisanship and other demographical variables such as race, gender, ethnicity and age, this study proposes a new paradigm via looking at online news sites as political communities where interactive content consumers create and consume the content in a self-fulfilling and sufficient way. Also, it is indicated that by and large political- content consumers online might be different in nature as they are not so much political-enthusiasm driven by political persuasions or grave social concerns and causes, as by those individuals' narcissistic psychological states, and that seeking a high level of public recognition might be a strong driver to political engagement online through various social platforms.

Therefore, this framework also can provide practical implications for news organizations that news consumption also combines with expressive participation to boost their knowledge and/or to gain recognition from peers in news-sites or social platforms. For instance, news organizations may benefit from rethinking how user comments are displayed and engaged with. That is, the "top" comment model (where the number of "up-voting" determines which comments receive "top" status, therefore is displayed at the top) may discourage active political 
participation among all users. A recent study (Oh, 2014) found that such an online discourse model potentially results in a misrepresentation of public opinion-"top" comments being displayed above others causes them to receive more "likes" and engagement, thus dictate the online discourse, especially in political issues. This is particularly pertinent for FOI characteristics studied in this article, as individuals with such traits may refrain from sharing their opinions when gauging the dominant opinion climate against their own. Thus, news organizations could consider restructuring commenting sections to create a more-welcoming environment for all voices. Such efforts can be seen in commenting sections of news websites such as NYT (providing options to view/sort comments in different ways, such as Editor's Picks), and Naver News (eliminating the "top" comment model for stories categorized under politics) (Park, 2018).

We also point out the importance of this study from an intercultural perspective. This study was conducted in South Korea, a culture and population that holds implications regarding key factors in our model. The study by Shim and Oh (2018) indicates that anonymity-seeking behavior is even stronger in South Korea due to its cultural characteristics. Such characteristics include individualism vs. collectivism from Hofstede's (1983) cultural dimensions and heightened influences of self-construal. According to the cultural dimensions perspective, Koreans (as part of the East Asian culture) display higher levels of collectivism as opposed to the individualism of the west. That is, Koreans value harmony and blending in as a whole, and refrain from actions of conversation that might be deemed different. By the same token, Koreans tend not to voice their opinions conspicuously, especially when they are recognizable, lest they cause discord among communities and collectives of which they are part (Choi \& Park, 2014).

Self-construal refers to the degree of an individual's concerns regarding how the self is construed by others. Markus and Kitayama (1991) notes that Eastern and Western individuals have "strikingly different construals of the self" (p. 224) in that individuals in Asian cultures define the self closely based on how others might view them, with emphasis on fitting in. In similar light, Yoo's (2012) study found that Koreans paid close attention to how others would view them, and even hesitated to publicly post their thoughts on social media due to concerns over how they will be judged by others. We believe that such characteristics increase anonymity-seeking inclinations among Koreans more so than in Western culture, where individuals participate in political conversations and voice opinions more freely. Thus, future studies could benefit from further exploring and online political discourse behavior in light of cultural differences between East and West.

\section{Limitations and Suggestions for Future Studies}

While this exploratory study aimed at a new approach to contribute to the scholarship on FOI and political discourse, we acknowledge that the article is not without limitations.

One limitation relates to measures of fear of isolation. FOI may manifest itself in different forms for individuals with different backgrounds. To be specific, FOI has differentiating subtle nuances in its concept such as trait-, issue-, and state-based fear. We measured only communication apprehension based on personal trait in this study, whereas future effort can explore different types of fear arising via a specific issue or personal situations. In that way we can examine how political expression via online outlets are connected to the various types of FOI whether source of fear derives from personal history of negative interactions or from a perception of dominant opinion about a specific issue, or from factors sensitive and unique to the political itself. Therefore, we believe that a refined definition and exploration of FOI itself based on online and political contexts is necessary.

We are concerned about methodology as our data lumped news consumption and contribution with the online conversation. While a big difference exists between reading news and contributing to online discussion, we are attentive to the fact that online news service is designed to simultaneously trigger consumption and participation via comment thread service, and it would be more convenient to recall the amount of time online as this survey relies on self-report based on personal memory. To address this limitation, future study might attempt to look at how frequency of online news consumption and participation can be related distinctively to FOI and online anonymity. Also we exclude above 60 age bracket in the survey who are active online users in Korean context, therefore, future study can collect representative sample for further exploration.

Also, in this study we looked at only six major social platforms for political engagement yet, nowadays, various platforms such as podcast and political communities have arisen as political agenda-setting and forming conversations, so future research could delve into more diverse and cutting-edge political communities to understand political expressions via online. Another limitation could stem from the fact that data was collected only in South Korea. While South Korea is known to be highly advanced in terms of Information Communication Technologies (ICTs), and boasts one of the highest numbers of Internet users, its media environment differs from those in other societies due to political and cultural discrepancies. Therefore, different online media environments may exert varying levels of influence on someone with FOI. Even with the same level of FOI, societal characteristics, online culture, and available resources may promote or inhibit behaviour similar to that of Korean participants.

\section{Acknowledgments}

The authors would like to thank anonymous reviewers for their valuable feedback and suggestions. 


\section{Conflict of Interests}

The author sdeclare no conflict of interests.

\section{References}

Bailenson, J. N., Yee, N., Merget, D., \& Schroeder, R. (2006). The effect of behavioral realism and form realism of real-time avatar faces on verbal disclosure, nonverbal disclosure, emotion recognition, and copresence in dyadic interaction. Presence, 15, 359-372.

Bargh, J. A., McKenna, K. Y. A., \& Fitzsimons, G. M. (2002). Can you see the real me? Activation and expression of the "true self" on the Internet. Journal of Social Issues, 58, 33-48.

Baron, R. M., \& Kenny, D. A. (1986). The moderatormediator variable distinction in social psychological research: Conceptual, strategic, and statistical considerations. Journal of Personality and Social Psychology, 51(6), 1173.

Bennett, W. L., \& lyengar, S. (2008). A new era of minimal effects? The changing foundations of political communication. Journal of communication, 58(4), 707-731.

Berg, J. (2016). The impact of anonymity and issue controversiality on the quality of online discussion. Journal of Information Technology \& Politics, 13(1), 37-51.

Berger, J. (2014). Word of mouth and interpersonal communication: A review and direction for future research. Journal of Consumer Psychology, 24(4), 586-607.

Chang Center Group. (2017). Portal site rankings. Retrieved from https://m.blog.naver.com/hb0675/ 220929640951

Chiou, W.-B. (2006). Adolescents' sexual self-disclosure on the Internet: Deindividuation and impression management. Adolescence, 41, 547-561.

Chiou, W.-B. (2007). Adolescents' reply intent for sexual disclosure in cyberspace: Gender differences and effects of anonymity and topic intimacy. CyberPsychology \& Behavior, 10, 725-728.

Cho, D., \& Kwon, K. H. (2015). The impacts of identity verification and disclosure of social cues on flaming in online user comments. Computers in Human Behavior, 51, 363-372.

Choi, S., \& Park, H. W. (2014). An exploratory approach to a Twitter-based community centered on a political goal in South Korea: Who organized it, what they shared, and how they acted. New Media and Society, 16(1), 129-148. doi:10.1177/1461444813487956

Coe, K., Tewksbury, D., Bond, B. J., Drogos, K. L. Porter, R. W., Yahn, A., \& Zhang, Y. (2008). Hostile news: Partisan use and perceptions of cable news programming. Journal of Communication, 58, 201-219.

DeWall, C. N., Buffardi, L. E., Bonser, I., \& Campbell, W. K. (2011). Narcissism and implicit attention seeking: Evidence from linguistic analyses of social networking and online presentation. Personality and Individual Differences, 51(1), 57-62.

Enli, G. (2017). Twitter as arena for the authentic outsider: Exploring the social media campaigns of Trump and Clinton in the 2016 US Presidential election. European Journal of Communication, 32, 50-61.

Fu, W. W., \& Sim, C. C. (2011). Aggregate bandwagon effect on online videos' viewership: Value uncertainty, popularity cues, and heuristics. Journal of the American Society for Information Science and Technology, 62(12), 2382-2395.

Gearhart, S., \& Zhang, W. (2014). Gay bullying and online opinion expression: Testing spiral of silence in the social media environment. Social science computer review, 32(1), 18-36.

Hayes, A. F., Matthes, J., \& Eveland, W. P. (2011). Stimulating the quasi-statistical organ: Fear of social isolation motivates the quest for knowledge of the opinion climate. Communication Research, 40(4), 439-462.

Hofstede, G. (1983). National cultures in four dimensions: A research-based theory of cultural differences among nations. International Studies of Management \& Organization, 13(1/2), 46-74.

Houston, J. B., Hansen, G. J., \& Nisbett, G. S. (2011). Influence of user comments on perceptions of media bias and third-person effect in online news. Electronic News, 5(2), 79-92.

Huberman, B. A., Romero, D. M., \& Wu, F. (2009). Crowdsourcing, attention and productivity. Journal of Information Science, 35(6), 758-765.

Jensen, M. J. (2017). Social media and political campaigning. International Journal of Press/Politics, 22(1), 23-42.

Joinson, A. N. (2001). Self-disclosure in computermediated communication: The role of self-awareness and visual anonymity. European Journal of Social Psychology, 31, 177-192.

Joinson, A. N. (2003). Understanding the psychology of internet behaviour: Virtual worlds, real lives. New York, NY: Palgrave Macmillan.

Joinson, A. N. (2007). Disinhibition and the Internet. In J. Gackenbach (Ed.), Psychology and the internet: Intrapersonal, interpersonal and transpersonal implications (2nd ed., pp. 76-92). San Diego, CA: Elsevier Academic Press.

Kiesler, S., Siegel, J., \& McGuire, T. W. (1984). Social psychological aspects of computer-mediated communication. American Psychologist, 39, 1123-1134.

Kim, S.-H., Kim, H., \& Oh, S.-H. (2014). Talking about genetically modified (GM) foods in South Korea: The role of the Internet in the spiral of silence process. Mass Communication and Society, 17(5), 713-732. doi:10.1080/15205436.2013.847460

Kim, S. (2010, March 15). 'MB Dokdo Statement Article' surpasses $100 \mathrm{~K}$ comments. Media Today. Retrieved from http://www.mediatoday.co.kr/?mod= news\&act $=$ articleView\&idxno $=86606$ 
Kim, S. H. (2012). Testing fear of isolation as a causal mechanism: Spiral of silence and genetically modified (GM) foods in South Korea. International Journal of Public Opinion Research, 24(3), 306-324.

Lampel, J., \& Bhalla, A. (2007). The role of status seeking in online communities: Giving the gift of experience. Journal of Computer-Mediated Communication, 12(2), 434-455.

Lapidot-Lefler, N., \& Barak, A. (2012). Effects of anonymity, invisibility, and lack of eye-contact on toxic online disinhibition. Computers in Human Behavior, 28, 434-443.

Lim, G. (2016, December 3). 6th weekend candlelight vigil sets new record: Organizers state '2.12 million'. Yonhap News. Retrieved from http://www. yonhapnews.co.kr/bulletin/2016/12/03/0200000000 AKR20161203058500004.HTML

Liu, X., \& Fahmy, S. (2011). Exploring the spiral of silence in the virtual world: Individuals' willingness to express personal opinions in online versus offline settings. Journal of Media \& Communication Studies, 3(2), 45-47.

Markus, H. R., \& Kitayama, S. (1991). Culture and the self: Implications for cognition, emotion, and motivation. Psychological Review, 98(2), 224-253.

McElroy, K. (2013). Where old (gatekeepers) meet new (media). Journalism Practice, 7(6), 755-771. doi:10.1080/17512786.2013.774117

Messing, S., \& Westwood, S. J. (2014). Selective exposure in the age of social media: Endorsements trump partisan source affiliation when selecting news online. Communication Research, 41(8), 1042-1063.

Ministry of the Interior (2015, July), Statistics of resident registration. Retrieved from http://rcps.egov. go.kr:8081/jsp/stat/ppl_stat_jf.jsp

Moe, W. W., \& Schweidel, D. A. (2012). Online product opinions: Incidence, evaluation, and evolution. Marketing Science, 31(3), 372-386.

Nagy, P., \& Koles, B. (2014). The digital transformation of human identity: Towards a conceptual model of virtual identity in virtual worlds. Journal of Research into New Media Technologies, 20(3), 276-292.

Naver. (2017). User comment rankings. Retrieved from http://m.news.naver.com/memoRankingList.nhn

Neuwirth, K., Frederick, E., \& Mayo, C. (2007). The spiral of silence and fear of isolation. Journal of Communication, 57, 450-468. doi:10.1111/j.14602466.2007.00352.x

Nielsen, C. (2014). Coproduction or cohabitation: Are anonymous online comments on newspaper websites shaping news content? New Media \& Society, 16(3), 470-487. doi:10.1177/1461444813487958

Nielsen KoreanClick. (2018). Internet usage trends in January 2018. Retrieved from http://www.koreanclick. com/insights/newsletter_view.html?code=trend\&id $=467 \&$ page $=1$

Noelle-Neumann, E. (1974). The spiral of silence: A theory of public opinion. Journal of Communication,
24(1), 24-51.

Oh, S. (2014). What's in a "like"? Influence of news audience engagement on the deliberation of public opinion in the digital public sphere (Unpublished doctoral dissertation), University of Maryland, MD.

Ott, B. L. (2017). The age of Twitter: Donald J. Trump and the politics of debasement. Critical Studies in Media Communication, 34(1), 59-68.

Papacharissi, Z. (2004). Democracy online: Civility, politeness, and the democratic potential of online political discussion groups. New media \& society, 6(2), 259-283.

Park, T. (2018, October 22). Naver news comment policies to be determined by news organizations. Hankyoreh. Retrieved from http://www.hani.co.kr/arti/ economy/it/866857.html

Podbbang. (2017). Podcast listenership ranking. Retrieved from http://www.podbbang.com/ranking/ daily

Porten-Cheé, P., \& Eilders, C. (2015). Spiral of silence online: How online communication affects opinion climate perception and opinion expression regarding the climate change debate. Studies in Communication Sciences, 15, 143-150.

Preacher, K. J., \& Hayes, A. F. (2008). Asymptotic and resampling strategies for assessing and comparing indirect effects in multiple mediator models. Behavior Research Methods, 40(3), 879-891.

Reuters. (2016). Digital news report 2016. Retrieved from http://www.digitalnewsreport.org

Shim, K., \& Oh, S. (2018). Who creates the Bandwagon? The dynamics of fear of isolation, opinion congruency and anonymity-efficacy on social media in the 2017 South Korean presidential election. Computers in $\mathrm{Hu}$ man Behavior, 86, 181-189.

Springer, N., Engelmann, I., \& Pfaffinger, C. (2015). User comments: Motives and inhibitors to write and read. Information, Communication \& Society, 18(7), 798-815.

Statista. (2016). Daily internet usage rate in South Korea in 2016, by age group. Statista. Retrieved from https://www.statista.com/statistics/348188/daily-in ternet-usage-age-group-south-korea

Suler, J. (2004). The online disinhibition effect. Cyberpsychology \& Behavior, 321-326.

Velasquez, A. (2012). Social media and online political discussion: The effect of cues and information cascades on participation in online political communities. New Media and Society, 14(8), 1286-1303.

Weiman, A. C., Fernandez, K. C., Levinson, C. A., Augustine, A. A., Larsen, R. J., \& Redebaugh, T. L. (2012). Compensatory internet use among individuals higher in social anxiety and its implications for well-being. Personality and Individual Differences, 53, 191-195.

Yoo, J. (2012). A qualitative analysis of how and why people use social network sites: A cross-cultural comparison of Korea and the U.S. Journalism and Mass Communication, 2(6), 1-17. 
Yun, G. W., \& Park, S.-Y. (2011). Selective posting: Willingness to post a message online. Journal of
Computer-Mediated Communication, 16, 201-227. doi:10.1111/j.1083-6101.2010.01533.x

\section{About the Authors}

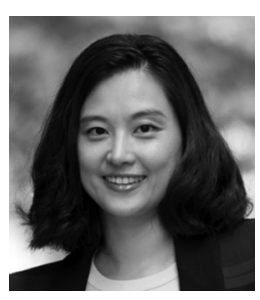

KyuJin Shim is a Lecturer in Media and Communications at the University of Melbourne. Prior to joining the University of Melbourne in 2018, KyuJin was an Assistant Professor of Corporate Communication at Singapore Management University, Singapore. KyuJin's research interests are ethical consumerism and political consumerism in the context of corporate ethics and social responsibility, and social media, crisis management and international PR with regard to globalization and digitization in communication.

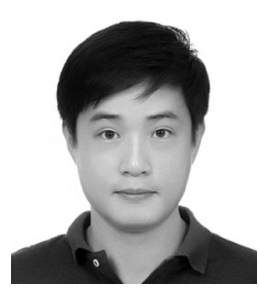

Klive (Soo-Kwang) Oh is an Assistant Professor at the Communication Division at Pepperdine University. His research interests include digital culture, new/social media and audience engagement. He has several research publications and awards regarding his work on various aspects of new media, public relations and journalism. He received his MA from the Missouri School of Journalism and PhD from University of Maryland. Before devoting himself to academia, he worked at Samsung Electronics. 


\section{Appendix 1}

\section{Fear of isolation (Kim, 2012)}

- I worry about being negatively evaluated when others disagree with me.

- I tend to let the other person win an argument even when I know I am right.

- If my political opinion were to become widely known around your workplace, I am very concerned about that people would avoid me or act differently toward me somehow.

\section{Attention/status-seeking (Berger, 2014)}

- I want to be perceived positively

- I would like to look special

- I would like to be considered an expert

- I would like to be considered more knowledgeable

- I would like to be seen as an opinion leader

- I would like to receive attention from others

\section{Anonymity-seeking (Self-developed items)}

- During anonymous online conversation via social media or online news site, I feel less constrained to talk about certain things than I would in identifiable conversation.

- The anonymous online platform enables me to support my favorite politicians on political news websites

- Thanks to anonymity online, I can argue without reserve with someone having a different opinion, than in the offline context

- The anonymous online platform enables me to engage in political discussion.

\section{Social Media Use (Self-developed items)}

- The next set of questions asks about your use of media for searching for news on politics and political conversations. Please provide your answer in ten-minute intervals (from 0 to 60+). Usually, on a typical day, how many minutes a day do you spend on these media platforms for political news consumption and conversation?
1) Facebook
2) Twitter
3) Naver news
4) Daum news
5) YouTube
6) Other online platforms 